\title{
Neurological and Cardiovascular Adverse Events Associated with Antimanic Treatment in Children and Adolescents
}

\author{
Jeanette M. Jerrell \\ University of South Carolina School of Medicine, Columbia, SC, USA
}

\author{
Keywords \\ Adolescents; Cardiovascular adverse events; \\ Children; Mood stabilizers; Neurological \\ adverse events.

\section{Correspondence} \\ Jeanette M. Jerrell, Ph.D., Department of \\ Neuropsychiatry and Behavioral Science, \\ University of South Carolina School of Medicine, \\ 3555 Harden Street Ext., 15 Medical Park Suite \\ 301, Columbia, SC 29203, USA. \\ Tel.: 803-434-4507; \\ Fax: 803-434-4277; \\ E-mail: Jeanette.Jerrell@uscmed.sc.edu
}

doi: 10.1111/j.1755-5949.2009.00087.x

Data analysis was supported by a State Mental Health Data Infrastructure Grant (SAMHSA SM54192).
To identify the factors associated with incident neurological and cardiovascular adverse events in children and adolescents treated with antimanic agents, a retrospective, longitudinal study was conducted. Medicaid medical and pharmacy claims between January 1996 and December 2005 were used to identify 3657 children and adolescents prescribed antimanic medications, and a random sample of 4500 children not treated with psychotropic medications. All adverse events examined (sedation/drowsiness, headaches, involuntary movements/extrapyramidal symptoms (EPS), cardiovascular events, hypertension, and orthostatic hypotension) were more prevalent in the antimanic-treated cohort. The odds of developing incident sedation/drowsiness and headaches were significantly higher for those prescribed carbamazepine, and co-prescribed selective serotonin reuptake inhibitors or antipsychotics. The odds of incident involuntary movements/EPS were significantly higher for those co-prescribed antimanic and antipsychotic agents, and those with comorbid central nervous system (CNS), organic brain disorders/mental retardation, or epilepsy. Incident cardiovascular events, hypertension, and orthostatic hypotension odds were significantly higher for those co-prescribed antimanic agents and antipsychotics, or those with comorbid epilepsy or metabolic conditions. Coprescription of antimanic and antipsychotic agents is more likely associated with neurological and cardiovascular adverse reactions, especially in young patients with preexisting CNS/neurological disorders.

\section{Introduction}

For complex mood disorder presentations in children and adolescents, for example, bipolar disorder, monotherapy with lithium, two anticonvulsants used as mood stabilizers (valproic acid derivatives and carbamazepine), or three second-generation antipsychotics (olanzapine, quetiapine, or risperidone) is considered to be first-line treatment, with augmentation by another mood stabilizer or second-generation antipsychotic if the firstline treatment is only partially effective [1]. Moreover, combinations of medications are increasingly prescribed for children with bipolar disorder and co-occurring conditions, e.g., attention-deficit hyperactivity disorder. However, because long-term side effects are more likely to occur and to occur more frequently with co-prescribed psychotropics [1], it is important to monitor the side effects associated with different prescribing patterns over time.
In this investigation, the incidence/prevalence of several neurological and cardiovascular adverse events in an antimanic-treated cohort from South Carolina's Medicaid system are compared with a random sample of children served through Medicaid with no exposure to psychotropic medications, in order to identify the factors related to these events, for example, preexisting medical conditions, the co-prescription of other psychotropic medications, and individual risk factors of age, gender, and race.

\section{Methods}

\section{Cohort Selection}

Claims data for South Carolina's Medicaid program were obtained through the state's Office of Research and Statistics. Each Medicaid medical claim identifies a service encounter and gives the date of service, and the International Classification of Diseases (ICD), Ninth Revision, 
Clinical Modification diagnosis codes related to that visit (Visit file). Pharmacy claims identified the medication dispensed and the date the prescription was filled (Pharmacy file). A separate data file regarding eligibility was used to summarize the demographics for each patient (Person file). The databases are frequently updated prior to being made available for analysis. This study was approved by the University of South Carolina Institutional Review Board as exempt from human subject research guidelines under 45 Code of Federal Regulations, part 46.

Medical and pharmacy claims for the calendar years January 1, 1996, through December 31, 2005, were used to identify a cohort of child and adolescent patients (ages $\leq 17$ ) enrolled in and eligible for Medicaid for a minimum of 9 months in each calendar year included in this analysis, who had a service encounter, and who were prescribed any of three medications used as mood stabilizers between January 1, 1998, and December 31, 2003. The date of first prescription of an antimanic/mood-stabilizing medication (i.e., valproic acid derivatives, lithium, and carbamazepine) in the Medicaid data set was defined as the selection encounter date. This process resulted in the identification of 3657 patients for the treated cohort.

Out of the same population and from the same period, medical and pharmacy claims were also used to identify a randomly selected group of child and adolescent patients (0-17 years old) eligible for Medicaid 9 out of 12 months in all calendar years under study, who had service encounters, but no prescriptions in the database for any class of psychotropic medication (antipsychotics, antidepressants, mood stabilizers, or psychostimulants). This process resulted in the identification of 40,660 patients who met the criteria. From this group, a random sample of 4500 patients was selected to use as a representative control group. $i$

\section{Adverse Event Coding}

Cardiovascular or neurological medical conditions that were detected in the 24 months prior to each patient's selection date into the treated cohort were coded as "preexisting" for this study. If the patient developed a medical condition subsequent to the prescription of the antimanic medication, new variables were created for these "incident" events. In the control group, detection of any of the cardiovascular or neurological medical conditions in a service billing record was coded. The following categories of conditions and events were evaluated in both the treated cohort and the control sample: cardiovascular events (ICD-9 codes: myocardial infarction 410-412; ischemic/pulmonary heart disease 413-416, 428-429; arrhythmias 426-427; and cardiomyopathy 425), cerebrovascular events (ICD-9 codes: cerebrovascular disease 436-437; cerebrovascular accident 435; cerebrovascular hemorrhage 430434; and peripheral vascular disease 440-448), orthostatic hypotension/syncope (ICD-9 codes: 458, 780.2), hypertension (ICD-9 codes: 401-405), involuntary movements/extrapyramidal symptoms (EPS) (including neuroleptic malignant syndrome) (ICD-9 codes: 333 to 333.99, 781.0), headaches (ICD-9 codes: 784.0), and sedation/somnolence/drowsiness (ICD-9 codes: 780.09, 780.54, 780.89).

For the cardiovascular adverse event analyses, preexisting congenital heart defects were coded as ICD-9: 747.0-747.9; comorbid type 2 diabetes mellitus and dyslipidemia were coded as ICD-9: 272, 288.0, 285.9 and $250,250.00-251.92$ with 5 th digit $=0,2$; and a substancerelated disorder was coded as ICD-9: 304 and 305. Comorbid medical conditions for the neurological adverse events analyses were coded as preexisting epilepsy (ICD9 codes: 340-345.9), preexisting central nervous system disorders (ICD-9 codes: 320-389.9), preexisting organic brain damage and moderate to profound mental retardation (ICD-9 codes: 310.x, 318.x), preexisting cardio- or cerebrovascular conditions, or comorbid obstructive sleep apnea (ICD-9 codes: 770.82 or 780.57 ).

\section{Statistical Analysis}

To address research questions regarding differences in the incidence/prevalence of the cardiovascular or neurological conditions/events in the treated cohort versus control sample, six multiple logistic regression equations were constructed to assess the relative odds associated with developing each adverse event, using the control sample as the primary comparator, and controlling for three individual risk factors (i.e., gender, ethnicity, and age), dichotomously coded as male/female, African American/other, and age 12 or less/age 13 or more.

Then, to identify factors associated with the cardiovascular or neurological events in the treated cohort of pediatric patients prescribed antimanic agents, including the role of comorbid medical conditions and coprescribed psychotropic medications on the development of these conditions, five separate multiple logistic regression equations (one combining cardio- and cerebrovascular events) were constructed to assess the relative odds associated with developing each adverse event under scrutiny, using the lithium and carbamazepine as the main antimanic agent covariates (compared to valproic acid derivatives), and co-prescriptions of selective serotonin reuptake inhibitor (SSRI) antidepressants, psychostimulants, or antipsychotics as additional covariates of interest, controlling for three dichotomously coded individual risk factors (i.e., gender, ethnicity, and age). 
To each logistic regression equation for the cardiovascular equation, the presence of several comorbid conditions were added as independent variables: "comorbid type 2 diabetes mellitus and dyslipidemia," "congenital heart conditions," and a "substance-related disorder" signifying conditions shown in previous studies to pose additional risk factors for patients prescribed these medications [2]. To each logistic regression equation for the neurological equations, variables coded for the presence of several comorbid conditions were added as independent variables: "preexisting cardio- or cerebrovascular conditions," "comorbid organic brain damage/mental retardation," "comorbid central nervous system disorders," and "comorbid obstructive sleep apnea" (for sedation only) [3-6].

Co-prescribed antidepressants were coded as SSRIs for citalopram, escitalopram, fluoxetine, fluvoxamine, paroxetine, and sertraline [7]. Co-prescribed psychostimulants coded in the analyses were methylphenidate, dextroamphetamine, and amphetamine salts. Co-prescribed antipsychotics coded in the analyses were aripiprazole, ziprasidone, quetiapine, risperidone, olanzapine, or haloperidol.

\section{Results}

\section{Patients}

The antimanic-treated cohort was primarily male $(63 \%)$ and white $(43 \%)$, and being treated primarily for bipolar disorder (37\%), attention-deficit hyperactivity disorder $(65 \%)$, and conduct disorder $(45 \%)$, with comorbid psychotic $(20 \%)$ or depressive disorders/features $(24 \%)$. The mean age of the cohort was $9.6(\mathrm{SD}=4.1)$ years at the time of mood stabilizer initiation (selection date into the treated cohort) (Table S1). Valproic acid derivatives were prescribed to $83 \%$ of these children and adolescents, along with the newest antidepressant agents (serotonin norepinephrine reuptake inhibitor/heterocyclic compounds) (51\%), psychostimulants $(61 \%)$, and antipsychotics (46\%). Larger percentages of the treated cohort had diagnosed comorbid organic brain damage/mental retardation ( $16.4 \%$ vs. $3.6 \%$ in the control group), central nervous system disorders $(6.7 \%$ vs. $5.1 \%$ in the control group), epilepsy (36.2\% vs. $1.0 \%$ in the control group), obesity/weight gain (11.6\% vs. $8.6 \%$ in the control group), metabolic disruption (type 2 diabetes and dyslipidemia $13.5 \%$ vs. $10.8 \%$ in the control group), and obstructive sleep apnea (10.5\% vs. $1.9 \%$ in the control group).

The control sample demographics were somewhat different: $43.8 \%$ male, $75.7 \%$ African American, and 18.7\% Caucasian, with a mean age of 7.6 years at selection into the random sample. Although the clients in the antimanic-treated cohort were about 2 years older at selection into the cohort (start date of the antimanic medication) than those in the control sample, data were compiled on the treated cohort for 2 years prior to their selection date for analysis of the preexisting conditions, making their average age at start date in the data set comparable to that of the control group (7.6 years).

\section{Comparison of Treated Cohort and Untreated Control Sample}

The incidence/prevalence rates for the neurological and cardiovascular conditions in the treated cohort and untreated control sample are shown in Table S2. Table S3 presents statistical comparisons of the treated cohort and untreated control sample, controlling for individual risk factors (i.e., age, gender, and ethnic). The cohort treated with antimanic medications and other psychotropic agents was more likely to have been diagnosed with sedation/somnolence (odds ratio, OR $=3.23$ ), involuntary movements/EPS ( OR $=9.09)$, headaches $(\mathrm{OR}=1.59)$, cardiovascular events $(\mathrm{OR}=2.78)$, hypertension $(\mathrm{OR}=1.61)$, and orthostatic hypotension/syncope $(\mathrm{OR}=2.78)$.

\section{Treated Cohort Analyses}

The odds of developing sedation/somnolence/drowsiness (Table S4) were significantly higher for those prescribed carbamazepine $(\mathrm{OR}=1.48)$, and those co-prescribed SSRIs (OR $=1.46)$ and antipsychotics $(\mathrm{OR}=1.29)$. There were also differences in the risk of developing sedation/somnolence due to comorbid obstructive sleep apnea $(O R=2.06)$, controlling for the significant influence of individual risk factors, with females and adolescents being more likely to have incident sedation/somnolence $(\mathrm{OR}=1.61$; OR $=1.35)$.

The odds of developing involuntary movements/EPS were significantly higher for those co-prescribed antipsychotics $(\mathrm{OR}=1.82 ; 95 \%$ confidence interval, $\mathrm{CI}=$ $1.22,2.72)$, and for those with preexisting central nervous system conditions $(\mathrm{OR}=2.25$; $\mathrm{CI}=1.22,4.13)$, organic brain syndromes or mental retardation $(\mathrm{OR}=2.35$; $\mathrm{CI}=1.64,3.37)$, and comorbid seizures $(\mathrm{OR}=2.19$; $\mathrm{CI}=1.37,3.50)$, with no antimanic medication group differences.

In the treated cohort, the odds of developing headaches were significantly higher for those prescribed carbamazepine ( $\mathrm{OR}=1.32$ ), and co-prescribed SSRI antidepressants $(\mathrm{OR}=1.22)$ and psychostimulant medications $(\mathrm{OR}=1.36)$, and females $(\mathrm{OR}=1.39)$ (Table $\mathrm{S} 5)$. 
Incident cardiovascular events were predominantly arrhythmias $(\mathrm{N}=169 ; 4.6 \%)$ and ischemic/pulmonary heart disease $(\mathrm{N}=77 ; 2.1 \%)$. The odds of developing cardio- and cerebrovascular events were higher for those co-prescribed antipsychotic medications $(\mathrm{OR}=1.55$; $\mathrm{CI}=1.12,2.13)$ regardless of the prescribed antimanic medications, and those with epilepsy $(\mathrm{OR}=1.93$; $\mathrm{CI}=$ $1.41,2.64)$, and comorbid metabolic disorders ( $\mathrm{OR}=2.16$; $\mathrm{CI}=1.53,3.07)$.

The odds of developing incident hypertension were significantly higher for children 13 years of age and older $(\mathrm{OR}=1.75$; $\mathrm{CI}=1.10,2.78)$, and those co-prescribed antipsychotics $(\mathrm{OR}=1.90 ; \mathrm{CI}=1.33,2.70)$ regardless of the prescribed antimanic medications, but significantly lower for those prescribed psychostimulants $(\mathrm{OR}=0.54$; $\mathrm{CI}=$ $0.38,0.76)$.

The odds of developing orthostatic hypotension/syncope were significantly higher for those taking SSRI antidepressants (OR $=1.32 ; \mathrm{CI}=1.01,1.73)$, those co-prescribed antipsychotics (OR = 2.19; $\mathrm{CI}=1.45,3.32)$ regardless of the prescribed antimanic medications, and those with comorbid epilepsy $(\mathrm{OR}=2.09$; $\mathrm{CI}=$ $1.40,3.12)$, controlling for the significant influence of individual risk factors, with females and adolescents being more likely to have incident orthostatic hypotension $/$ syncope $(\mathrm{OR}=1.40 ; \mathrm{CI}=1.10,1.79 ; \mathrm{OR}=1.70$; $\mathrm{CI}=1.16,2.49$, respectively).

\section{Discussion}

The results of this retrospective, longitudinal investigation indicate that, compared to youth not treated with any psychotropic medications, those treated with antimanic medications were significantly more likely to demonstrate all the neurological and cardiovascular adverse events examined: sedation/somnolence, involuntary movements/EPS, headaches, hypertension, orthostasis/syncope, and cardio- and cerebrovascular events. However, since many of these young patients had comorbid medical conditions that would predispose them to neurological and cardio-/cerebrovascular adverse events (i.e., epilepsy 36\%, central nervous system disorders $7 \%$, and organic brain/pervasive developmental disorders $16 \%$ ), and were in long-term treatment, during which they were exposed to multiple psychotropic medications, including antimanic, antipsychotic, and antidepressant agents, the higher prevalence of all neurological and cardiovascular conditions might be expected [1].

The main factors mitigating against the development of adverse events in this clinical context were the extensive use of valproic acid derivatives $(83 \%)$, as well as the use of newer antidepressant agents (serotonin nore- pinephrine reuptake inhibitor/heterocyclic compounds) $(51 \%)$, psychostimulants $(61 \%)$, and second-generation antipsychotics $(36 \%)$, all of which are associated with fewer or less severe neurological and cardiovascular adverse effects [1,7-23].

Those at significantly higher risk of incident sedation/somnolence were prescribed carbamazepine and coprescribed SSRIs and/or antipsychotics, as were those with preexisting obstructive sleep apnea or central nervous system disorders. Incident headaches were more likely to be reported for those prescribed carbamazepine and co-prescribed SSRIs. Incident involuntary movements/EPS (including neuroleptic malignant syndrome, since it could not be distinguished in the ICD codes) were found for those co-prescribed antipsychotics, as well as those with preexisting central nervous system, organic brain/mental retardation, and epilepsy/seizure disorders. The association between carbamazepine or co-prescribed antipsychotic or SSRI usage and neurological adverse events, such as sedation, involuntary movements/EPS, and headaches, comports with the results of several previous investigations $[7,9,10]$. Sedation/somnolence is more likely to develop in patients prescribed both lithium and an antipsychotic or SSRIs [7,24,25] or prescribed both valproate and quetiapine [8], but in this data set there were too few cases with those combinations of medications to statistically explore the effect of the medication interaction on each adverse event. The significant relationship between several preexisting central nervous system or organic brain conditions and a heightened risk of some neurological adverse effects, regardless of the psychotropic medication prescribed, also reflects some previous clinical trial results and case reports $[3,5,6,26]$. However, these findings provide additional evidence that children with certain pervasive developmental, central nervous system, or metabolic medical conditions are at increased risk for adverse events when exposed to multiple psychotropics.

A significantly higher risk for incident cardiovascular events was identified for those exposed to co-prescribed antipsychotics, as well as those with preexisting epilepsy and metabolic conditions. This higher risk of cardiovascular adverse events, notably arrhythmias and ischemic events, has been previously noted in clinical trials, case reports, and pharmacoepidemiologic studies $[1,2,9,10,19]$. The inability to detect an association between antimanic usage and hypertension in this analysis diverges from clinical trial studies, which have found a relationship between mood-stabilizing medications and hypertension [7]. However, an association between coprescribed antipsychotic agents and the development of hypertension, regardless of the antimanic agent prescribed, was identified. The age-related finding of an 
increased risk for hypertension in adolescents coprescribed mood-stabilizing and antipsychotic agents comports with a previous finding of increased risk of hypertension in adolescents predominantly prescribed antipsychotics over extended periods [2].

A significantly higher risk for incident orthostatic hypotension was found for those co-prescribed antipsychotics or SSRIs, but not for any particular antimanic agent, which partially diverges from previous findings. However, this result underscores the importance of knowing the relative safety profiles of the individual antimanic, SSRI, and antipsychotic agents, as well as understanding that there is an increased probability of adverse events associated with the co-prescription of these classes of medications $[1,9,10,19,27,28]$. Both psychiatric and primary care practitioners need to familiarize themselves with the potential for central nervous system toxicity associated with antimanic and antipsychotic agents in pediatric populations, and use them sparingly in children already displaying early-onset risk factors for neurological or cardiovascular adverse events $[2,3,5,9,10$, $28,29]$.

\section{Strengths and Limitations}

The perspective provided by the longitudinal database has several strengths:

(1) The cohort represents a large, heterogeneous group of children and adolescents with varying periods of exposure to antimanic agents and other psychotropics.

(2)There is sufficient power in the treated cohort and untreated sample sizes to detect somewhat lowincidence conditions and combine these conditions into related groupings.

(3) Previous studies have found that although Medicaid databases provide much less detailed information on individuals than a structured research interview, the physician diagnoses and utilization data are more reliable than client or family self-reports [30,31].

(4)The outcomes of disparate neurological and cardiovascular events related to antimanic and concomitant medication use are clinically relevant and of substantial public health importance in pediatric populations.

These results also need to be interpreted with several limitations in mind:

(1)The data were not based on controlled trial methods but, instead, we used secondary administrative data and observational techniques in a retrospective cohort design.
(2) Structured research and clinical interviews were not employed to confirm any of the assigned medical disorders.

(3)The reporting of adverse events was based on spontaneous reporting to a physician and is, consequently, likely to be an underestimate.

(4)The detection of any adverse event was also dependent on the ICD-9 codes being properly submitted to Medicaid and is also likely to be an underestimate.

(5)These results report associations and, as a result, directions of causality cannot be inferred.

(6)Key risk factors such as family history of specific cardiovascular disorders were not available in the database and are not modeled in these analyses.

(7)The representativeness of this Medicaid cohort in relation to those in other states and service systems cannot be estimated from our data set.

In order to provide a more refined estimate of the neurological or cardiovascular risk attributable to long-term exposure to antimanic agents or various co-prescribed antipsychotics or antidepressants, pragmatic and randomized, controlled efficacy, and effectiveness studies of antimanic therapy in combination with other psychotropic agents need to be conducted. Urgently needed, as well, are studies that draw further attention to the safety profile of antimanic agents in young populations and help develop an empirical foundation to guide clinical decision support, since there is only limited long-term safety information available regarding the use of these antimanic medications for pediatric patients. These results confirm findings from many previous short-term, clinical trial and case studies of the tolerability and safety of these antimanic agents, while demonstrating their development and prevalence in community-based practice settings, especially when multiple classes of psychotropic agents are co-prescribed. These findings are germane to the overall appraisal of the benefits and risks of this class of psychotropic agents. When evaluating the overall benefitrisk ratio of antimanic agents in children and adolescents, the practitioner needs to give careful consideration to a range of possible neurological and cardiovascular adverse reactions, especially in individuals receiving concomitant psychotropic medications, and to children with preexisting or comorbid epilepsy, or developmental/central nervous system disorders, which might further increase their risk of adverse reactions.

\section{Acknowledgment}

The author thanks Roger S. McIntyre, M.D., for helpful comments on an earlier version of the manuscript, and Ling Li, MSPH, and Maria Butkus, MPH, for performing the analyses presented herein. The views expressed do 
not necessarily represent those of the funding agency or official findings of the South Carolina Department of Health and Human Services (Medicaid).

\section{Conflict of Interest}

The author has no commercial associations that might pose a conflict of interest in the publication of this article.

\section{References}

1. Kowatch RA, Fristad M, Birmaher B, Wagner KD, Findling RL, Hellander M, Child Psychiatric Workgroup on Bipolar Disorder. Treatment guidelines for children and adolescents with bipolar disorder. J Am Acad Child Adolesc Psychiatry 2005;44:213-235.

2. McIntyre RS, Jerrell JM. Metabolic and cardiovascular events associated with antipsychotic treatment in children and adolescents. Arch Pediatr Adolesc Med 2008;162:929-935.

3. Devane CL, Sallee FR. SSRIs in child and adolescent psychiatry: A review of published experience. J Clin Psychiatry 1996;57:55-66.

4. Jerrell JM, Hwang TL, Livingston TS. Neurological adverse effects of antipsychotic use in children and adolescents. J Child Neurol 2008;23:1392-1399.

5. Magulac M, Landsverk J, Golshan S, Jeste DV. Abnormal movements in neuroleptic-naïve children and adolescents. Can J Psychiatry 1999;44:368-373.

6. Velamoor VR. Neuroleptic malignant syndrome: Recognition, prevention, and management. Drug Saf Concepts 1998;19:73-82.

7. Gold Standard, Inc. Carbamazepine, lithium, valproic acid derivatives, citalopram, escitalopram, fluoxetine, fluvoxamine, paroxetine, sertraline: adverse reactions. MD Consult [database] 2008. University of South Carolina School of Medicine Library, Columbia, SC. Accessed January 30, 2008.

8. Azorin JM, Findling RL. Valproate use in children and adolescents with bipolar disorder. CNS Drugs 2007;21:1019-1033.

9. Cheng-Shannon J, McGough JJ, Pataki C, McCracken JT. Second generation antipsychotic medications in children and adolescents. J Child and Adolesc Psychopharm 2004; 14:372-394.

10. Correll CU, Penzner JB, Parikh UH, Mughal T, Javed T, Carbon M, Malhotra AK. Recognizing and monitoring adverse events of second-generation antipsychotics in children and adolescents. Child Adolesc Psychiatric Clin N Am 2006;15:177-206.

11. Coulter DM, Pillans PI. Fluoxetine and extrapyramidal side effects. Am J Psychiatry 1995; 152:122-125.

12. Francis PD. Effects of psychotropic medications on pediatric electrocardiogram and recommendations for monitoring. Curr Opin Pediatr 2002; 14:224-

230.

13. Masi G, Mucci M, Pari C. Children with schizophrenia: Clinical picture and pharmacological treatment. CSN Drugs 2006;20:841-866.

14. McConville BJ, Sorter MT. Treatment challenges and safety considerations for antipsychotic use in children and adolescents with psychoses. J Clin Psychiatry 2004;65(Suppl 6):20-29.

15. Michelson D, Faries D, Wernicke J, Kelsy D, Kendrick K, Sallee R, Spencer T, Atomoxetine ADHD Study Group. Atomoxetine in the treatment of children and adolescents with attention-deficit/hyperactivity disorder: A randomized, placebo-controlled, dose-response study. Pediatrics 2001;108:E83.

16. MTA Cooperative Group: National Institute of Mental Health Multimodal Treatment Study of ADHD follow-up: Changes in effectiveness and growth after the end of treatment. Pediatrics 2004;113:762-769.

17. Skowron DM, Stimmel GL. Antidepressants and the risk of seizures. Pharmacotherapy 1992;12:18-22.

18. Stigler KA, Potenza MN, McDougle CJ. Tolerability profile of atypical antipsychotics in children and adolescents. Paediatr Drugs 2001;3: 927-942.

19. Wagner KD, Weller EB, Carlson GA, Sachs G, Biederman J, Frazier JA, Wosniak P, Tracy K, Weller RA, Bowden C. An open-label trial of divalproex in children and adolescents with bipolar disorder. $J$ Acad Child and Adol Psychiatry 2002;41:1224-1230.

20. Damsa C, Bumb A, Bianchi-Demicheli F, Vidailhet $P$, Sterck R, Andreoli A, Beyenburg S. “Dopamine dependent" side effects of SSRIs: A clinical review. J Clin Psychiatry 2004;65:1064-1068.

21. Hu XH, Bull SA, Hunkeler EM, Ming E, Lee JY, Fireman $B$, Markson LE. Incidence and duration of side effects and those rated as bothersome with SSRI treatment for depression: Patient report versus physician estimate. $J$ Clin Psychiatry 2004;65:959-965.

22. Masand PS, Gupta S. Long-term side effects of newer-generation antidepressants: SSRIs, venlafaxine, nefazodone, bupropion, and mirtazapine. Ann Clin Psychiatry 2002;14:175-182.

23. Pacher P, Kecskemeti V. Cardiovascular side effects of new antidepressants and antipsychotics: New drugs, old concerns? Curr Pharm Des 2004;10:2463-2475.

24. Gill J, Singh H, Nugent K. Acute lithium intoxication and neuroleptic malignant syndrome. Pharmacotherapy 2003;23:811-815.

25. Rosebush P, Stewart T. A prospective analysis of 24 episodes of neuroleptic malignant syndrome. Am J Psychiatry 1989;146:717-725.

26. Jensen PS, Buitelaar J, Pandina GJ, Binder C, Haas M. Management of psychiatric disorders in children and adolescents with atypical antipsychotics. Eur Child Adolesc Psychiatry 2007;16:104-120. 
27. Keck PE. The role of second-generation antipsychotic monotherapy in the rapid control of acute bipolar mania. $J$ Clin Psychiatry 2005;66(Suppl 3):5-1 1.

28. McIntyre RS, Konarski JZ. Tolerability profiles of atypical antipsychotics in the treatment of bipolar disorder. J Clin Psychiatry 2005;66(Suppl 3):28-36.

29. Ellsworth AJ, Witt DM, Dugdale DC, Oliver LM. Mosby's medical drug reference. St. Louis, MO: Mosby, 2006.

30. Holmberg SK, Kane C. Health and self-care practices of persons with schizophrenia. Psychiatr Serv 1999;50:827-829.

31. Mark TL, Buck JA, Dilonardo JD, Coffey RM, Chalk M. Medicaid expenditures on behavioral health care. Psychiatr Serv 2003;54:188-194.

\section{Supporting Information}

Additional Supporting Information may be found in the online version of this article:
Table S1 Descriptive analysis of the cohort of 3657 youths prescribed antimanic medications.

Table S2 Overall prevalence and incidence rates for pediatric patients prescribed antimanic medications and the control group.

Table S3 Comparison of prevalence of medical conditions in the antimanic-treated cohort and untreated control sample, controlling for individual risk factors.

Table S4 Adjusted odds ratios for incident sedation/somnolence.

Table S5 Adjusted odds ratios for incident headaches.

Please note: Wiley-Blackwell are not responsible for the content or functionality of any supporting materials supplied by the authors. Any queries (other than missing material) should be directed to the corresponding author for the article. 